\title{
BMJ Open Analysis of non-respondent pregnant women who were registered in the Japan Environment and Children's Study: a longitudinal cohort study
}

\author{
Mika Kigawa, ${ }^{\oplus 1}$ Akiko Tsuchida, ${ }^{2,3}$ Kayoko Miura, ${ }^{4}$ Mika Ito, ${ }^{5}$ Tomomi Tanaka, ${ }^{3,6}$ \\ Kei Hamazaki, ${ }^{2,3}$ Yuichi Adachi, ${ }^{6}$ Shigeru Saito, ${ }^{5}$ Hideki Origasa, ${ }^{7}$ \\ Hidekuni Inadera, ${ }^{2,3}$ Japan Environment and Children's Study (JECS)
}

To cite: Kigawa M, Tsuchida A, Miura K, et al. Analysis of non-respondent pregnant women who were registered in the Japan Environment and Children's Study: a longitudinal cohort study. BMJ Open 2019;9:e025562. doi:10.1136/ bmjopen-2018-025562

- Prepublication history for this paper is available online. To view these files, please visit the journal online (http://dx.doi. org/10.1136/bmjopen-2018025562).

Received 14 August 2018 Revised 22 May 2019 Accepted 23 May 2019
Check for updates

(C) Author(s) (or their employer(s)) 2019. Re-use permitted under CC BY-NC. No commercial re-use. See rights and permissions. Published by BMJ.

For numbered affiliations see end of article.

Correspondence to

Dr Mika Kigawa;

kigawa-c5s@kuhs.ac.jp

\section{ABSTRACT}

Objectives Non-response to questionnaires in a longitudinal study reduces the effective sample size and introduces bias. We identified the characteristics of nonrespondent pregnant women, and compared them with respondents in the Japan Environment and Children's Study (JECS) during the gestational period.

Design This was a questionnaire-based, Iongitudinal cohort study.

Setting Questionnaires were provided by research coordinators to mothers at prenatal examinations (at obstetrics clinics) or by mail. Mothers were measured twice: during the first trimester and during the second/ third trimester.

Participants Data were collected from the 10129 participating mothers of the 10288 children surveyed in the 2011 baseline JECS. We excluded responses from mothers who had a miscarriage or stillbirth; therefore, we analysed data from 9649 participants.

Primary and secondary outcome measures Data concerning demographics, medical history, health characteristics, health-related behaviour and environmental exposure were collected via selfadministered questionnaires. The response status of participants' partners and contact with their obstetrician were also examined. Multivariate logistic regression analysis was used to examine factors related to nonresponse.

Results Response was associated with living with one's mother-in-law (ORs: $0.47,95 \%$ Cls: 0.24 to 0.85 ), positive participation of participants' partner (OR: $0.25,95 \% \mathrm{Cl}$ : 0.17 to 0.35 ) and multiple visits to the obstetrician (OR: $0.02,95 \% \mathrm{Cl}: 0.02$ to 0.03 ). Participants who had a medical history of allergic rhinitis, had body pain or drank alcohol had higher odds of responding (ORs: 0.68, 0.96 and $0.36,95 \%$ Cls: 0.48 to 0.95 and 0.95 to 0.98 and 0.16 to 0.72 , respectively); those exposed to secondary smoke had lower odds of responding (OR: $1.59,95 \% \mathrm{Cl}: 1.12$ to 2.23).

Conclusions The non-response rate decreased when participants reported health-related behaviour or characteristics. Obtaining the understanding of people around each participant might help increase response rates.
Strengths and limitations of this study

- The Japan Environment and Children's Study is a nation-wide birth cohort study that includes 10129 mothers with confirmed obstetric outcomes in the first year of recruitment.

D During the gestational period, we provided self-administered questionnaires to mothers twice.

- The study is strengthened by its assessment of the effects of non-response on prevalence estimates as well as the exposure-outcome relationship.

- The sample size of this study was sufficient to examine the risk factors of non-response.

- We were unable to examine the effects of some socioeconomic factors on non-response.

\section{BACKGROUND}

Population-based studies are used to provide epidemiological data on the occurrence of disease and to identify risk factors that may be relevant to these outcomes. The Japan Environment and Children's Study (JECS) is a nation-wide birth cohort study that started recruiting expectant mothers in January $2011 .{ }^{1}$

In the first year of recruitment, approximately 10000 registered pregnant women had confirmed obstetric outcomes. Data on participants' health-related behaviour, marital status, socioeconomic status and education level were collected via self-administered questionnaires provided twice during the gestational period. ${ }^{2}$

In recent years, the response rates have decreased in several epidemiological studies over time. Although a particular study may achieve a high response rate, the prevalence estimates may still be biased if the non-responses are not random. The non-response bias may be related to selection bias; thus, the characteristics of non-respondents need to be 
confirmed. ${ }^{34}$ Systematic differences in the characteristics of respondents and non-respondents detract from the outcomes of interest. Therefore, the presence and extent of such bias should be investigated. ${ }^{5}$ In a cross-sectional health survey, Pietila and colleagues compared the backgrounds of responding and non-responding young men and found that their socioeconomic status and education level were related to their response status. ${ }^{6}$ Furthermore, the response status in the Atherosclerosis Risk in Communities Study differed according to sex and ethnicity. ${ }^{7}$

Long-term follow-up studies are hampered by a decrease in response rate due to the lapse of time between birth and follow-up. A systematic review of randomised controlled trials using postal questionnaires showed that the response rate was related to the length and/or design of questionnaire, use of personalised letters and follow-up contact, and matched the interests of participants and originating sources. ${ }^{8}$ In longitudinal cohort studies, various factors have been shown to be related to response status, including age, sex, marital status, education, health status, health-related behaviour, lifestyle, ethnicity, study objectives, contact modes, number and order of contact modes and use of incentives. ${ }^{9-12}$

Some authors have suggested that non-response increases the proportion of infants with adverse outcomes in the remaining study population ${ }^{13}$; however, how these factors influence study outcomes is unclear. Therefore, we performed this study to describe the characteristics of non-responders. We studied pregnant women who were registered in a prospective, cohort study and who did not return the second questionnaire during the gestational period.

\section{METHODS}

\section{Design of the JECS}

In the JECS, self-administered questionnaires were provided to mothers twice: during the first trimester (MT1) and during the second/third trimester (MT2). Questionnaires were provided by research coordinators at prenatal examinations (in the obstetrics clinic) or by mail and returned either by hand at subsequent prenatal visits (in the obstetrics clinic) or by mail. The partners of registered mothers were also asked to participate. We collected data from registered partners during the women's pregnancy through self-administered questionnaires returned by hand or by mail. Women's medical records were transcribed three times, by obstetricians, midwives/nurses or research coordinators at the obstetrics clinic: during the first trimester, during the second/ third trimester and after delivery.

\section{Design of the non-responder study}

In this study, we defined 'non-respondents' as JECS participants who did not return the questionnaire of second/ third trimesters. This study was based on a dataset (ie, jecs-ag-ai-20131008), which was released in October 2013. (The dataset supporting the conclusions of this article will be available after the steering committee of the JECS permits its accessibility.) The participant flow is illustrated in figure 1 .

Using the MT1 questionnaire, demographic data (age, marital status and cohabiting family members), medical and obstetric history, health-related behaviour (smoker/ exposure to secondary smoke and alcohol consumption) and occupational data were collected. The SF-8 Health Survey questionnaire (Japanese version) ${ }^{14}$ was used to assess participants' health-related quality of life (QOL). The K6 questionnaire (Japanese version) was used to assess participants' psychological distress. ${ }^{15}$ Age was divided into four categories: <25 years, 25-29 years, 30-34 years and $\geq 35$ years. We collected data of cohabiting family members via multiple-choice questionnaires.

The response data from participants' partners and a transcription sheet regarding health status data during the gestational period were linked with each participant.

\section{Definitions}

Participants' obstetric visiting status was a binary variable and was defined as present for a participant when the transcription sheet was returned if they had reported 'multiple obstetric visits to collaborating hospitals during pregnancy'. Partners' participation status was defined as positive when partners returned the questionnaire.

We collected information on occupation and types of employment of participants with the MT1 questionnaire. We focused on the following settings: homemakers or unemployed, worked from home and employed. For allocation of these settings, we used the Japan Standard Occupational Classification and the classification of positions in employment by the Ministry of Internal Affairs and Communication.

Regarding exposure to secondary smoke before pregnancy, 'daily' was defined as when participants answered with 'exposed at least once a week'.

\section{Patient and public involvement}

JECS started recruiting expectant mothers in January 2011 with the aim of assessing environmental factors that affect children's health, with the goal of providing a foundation for policymaking to safeguard the environment for the next generation. JECS study aimed to recruit approximately 100000 pregnant women and their partners over 3 years, to collect biological samples, and to collect data on their children until they turned 13 years old. ${ }^{1}$

Written informed consent for participation in JECS was obtained from individual mothers. In addition to the JECS main study, adjunct studies were conducted by the member of JECS group, or any combination of them. The adjunct studies may have included procedures that were not adopted by the main study, for example, collection and examination of placenta. This study was one of the adjunct studies of JECS, based on an existing dataset, and hence, patients were not directly involved in the sampling process. 


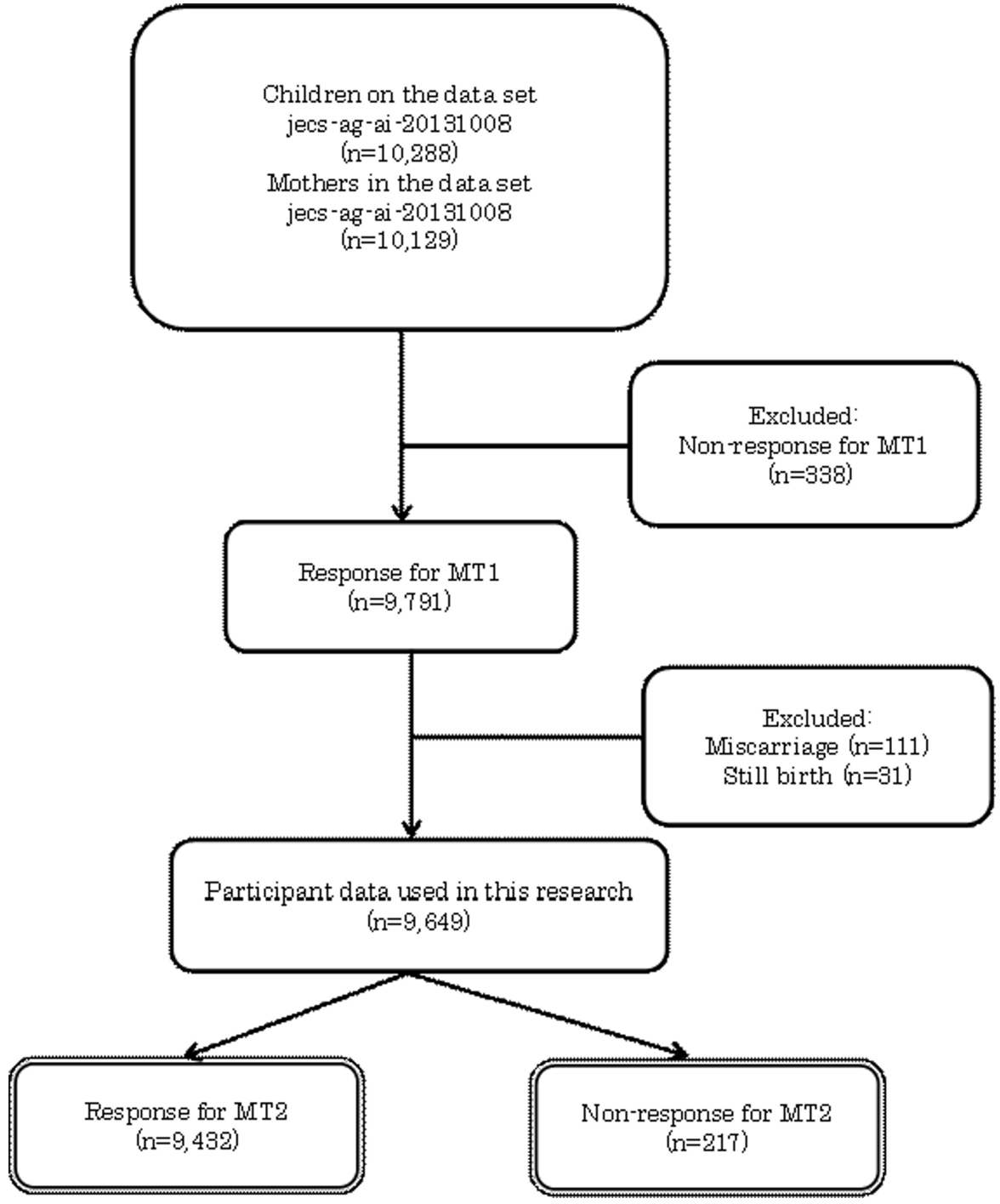

Figure 1 Participant (expecting mothers) flow.

\section{Ethical considerations}

The JECS protocol was reviewed and approved by the Ministry of the Environment's Institutional Review Board on Epidemiological Studies and by the Ethics Committees of all participating institutions. Written informed consent was obtained from all participating women and their partners.

\section{Statistical analyses}

The following variables were considered in the analyses for mothers: demographic data (age, marital status and cohabiting family members), medical and obstetric history, physical and mental health, health-related behaviours, occupation, environmental exposure, contact status with their obstetrician and partners' response status. Of these variables, a Student's t-test or Welch's t-test for independent groups was used for physical and mental health variables (SF-8, K6), or number of cohabiting family members (continuous variables), and a Pearson's $\chi^{2}$ test or Fisher's exact test was used for other variables (categorical variables). The variables that had significant associations with non-response to the MT2 questionnaire in the bivariate logistic regression models were included in the multivariate models. Prevalence ORs and 95\% CIs for non-response were estimated using multivariate logistic regression analyses. The contribution of a variable to the regression model was assessed using the likelihood ratio test.

A significance level of 0.05 (two-tailed) was used for all statistical tests. JMP Pro V.11 (SAS Institute, Cary, NC, USA) was used for all statistical analyses.

\section{RESULTS}

The overall response rate to the questionnaire in the second/third trimester was $97.7 \%$ (9432/9649). Table 1 shows the participants' characteristics at the first trimester, their partners' participating status and visits to the obstetrician among responders and non-responders. The proportions of marital status, family members, medical history, exposure to secondary smoke and job status significantly differed between responders and non-responders. The responders were more likely to be married, living with in-laws, have a history of allergic 
Open access

Table 1 Baseline characteristics of sample

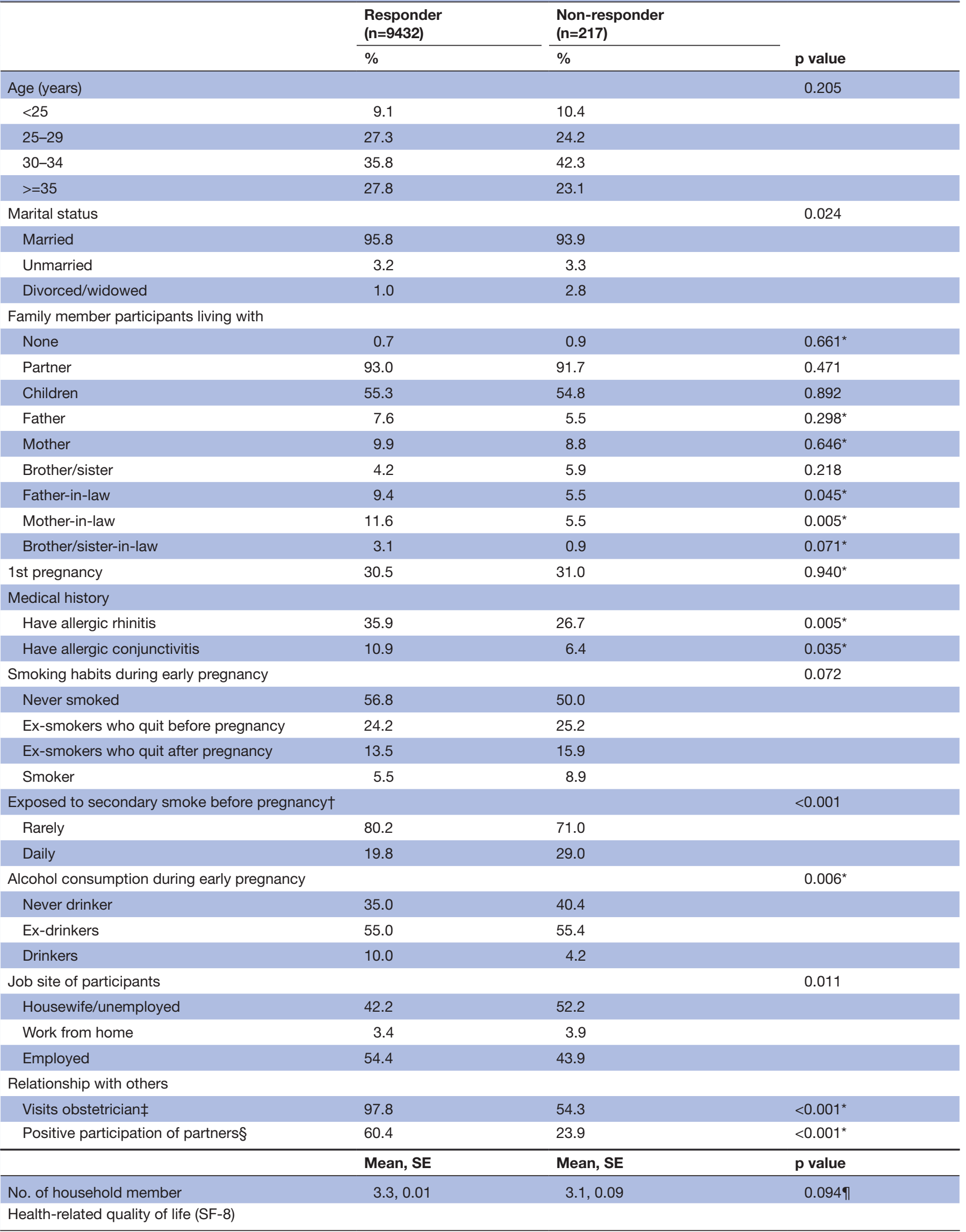

Continued 
Table 1 Continued

\begin{tabular}{llll}
\hline & Mean, SE & Mean, SE & p value \\
\hline General health & $46.9,0.1$ & $46.7,0.5$ & 0.772 \\
\hline Physical functioning & $46.6,0.1$ & $45.5,0.5$ & 0.027 \\
\hline Role physical & $43.7,0.1$ & $43.5,0.6$ & 0.756 \\
\hline Body pain & $50.0,0.1$ & $48.7,0.6$ & 0.025 \\
\hline Vitality & $47.5,0.1$ & $47.2,0.5$ & 0.452 \\
\hline Social functioning & $44.2,0.1$ & $43.4,0.6$ & 0.203 \\
\hline Mental health & $47.0,0.1$ & 0.062 \\
\hline Role emotional & $47.2,0.1$ & 0.198 \\
\hline Physical component summary & $45.5,0.1$ & $46.2,0.5$ & 0.203 \\
\hline Mental component summary & $46.3,0.1$ & $46.5,0.5$ & 0.164 \\
\hline Self-administered mental health (K6) & $9.6,0.0$ & $44.8,0.5$ & 0.0979 \\
\hline
\end{tabular}

${ }^{\star}$ Fisher's exact test.

†'Daily' defined as subjects exposed at least once a week.

$\ddagger$ Participants who collected the transcription sheet defined as multiple visits with obstetrician.

§Positive participation of partner was those who answered the questionnaire.

ףWelch's t-test.

rhinitis or allergic conjunctivitis, have better physical functioning, have a high response rate from their partner and make more visits to the obstetrician. Additionally, responders were less likely to have a history of migraines or polycystic ovary syndrome than were non-responders. Non-responders were more likely to have been exposed to secondary smoke than were responders. Participants who were employed were more likely to respond than were their counterparts. The SF-8 physical functioning and body pain scales were significantly higher for responders than for non-responders.

Two variables showed significant associations-living with one's mother-in-law and having allergic rhinitiswith non-response according to the bivariate logistic regression model. Tables 2A,B shows the ORs for non-response according to the various demographic and clinical characteristics, partners' participation status and visiting obstetricians in the multivariate logistic regression analyses. Model 1 included the variables that had significant associations with non-response of MT2.

The odds of non-response were lower in participants who had a medical history of allergies, which is one of the priority outcomes of the JECS ${ }^{1}$; had a positive QOL; were living with their mother-in-law; had partners who actively participated and had maintained contact with obstetricians. However, the odds of non-response were higher in participants who had been exposed to secondary smoke. Marital status, job site and the SF-8 physical functioning scale did not match the model, and thus were excluded.

Model 2 excluded variables that did not show significance in model 1 . The odds of non-response were higher in participants who had been exposed to secondary smoke; however, the odds were lower in participants who lived with their mother-in-law, had a history of allergic rhinitis, had a positive QOL regarding body pain, had partners who participated and visited the obstetrician.

\section{DISCUSSION}

Using data collected during pregnancy, we evaluated non-response bias in approximately 10000 pregnant women who participated in the JECS. Many factors were independently associated with response to the follow-up questionnaire. The characteristics associated with a greater probability of response included being married, living with one's mother-in-law and where the participants worked. Having a medical history of allergic rhinitis or allergic conjunctivitis resulted in a higher probability of response. The number of partners with positive participation in the JECS and multiple visits to the obstetrician were significantly lower in non-responders than in responders.

The ORs for non-response were correlated with demographic and clinical characteristics, partners' participation status and visiting the obstetrician in the multivariate logistic regression analysis. Specifically, the odds of non-response were lower in participants who had a medical history of allergies, which is one of the priority outcomes of the JECS; who had a positive QOL; who were living with their mother-in-law; whose partners participated and who maintained contact with obstetricians. The odds of non-response were higher in participants who had been exposed to secondary smoke. Baron and colleagues reported that passive smoking showed disparity across educational levels. ${ }^{16}$ We could not consider the effects of education; however, the relationship between non-response and exposure to secondary smoke might be affected by participants' education.

One of the objectives of the JECS was to assess environmental factors that affect children's health (eg, allergic diseases). The prevalence of allergic rhinitis in the Japanese population was $44.2 \%$ in $2006-2007^{17}$ and that of allergic conjunctivitis disease was $14.8 \%$ in $1993 .{ }^{18}$ Both were higher than those reported in the current study 
Table 2A Multivariate logistic regression predicting the likelihood of survey non-response: model 1

\begin{tabular}{|lll}
\hline Variable & OR (95\% Cl) & p value \\
\hline Marital status & & \\
\hline Married & Reference & \\
\hline Unmarried & $0.64(0.23$ to 1.48$)$ & 0.324 \\
\hline Divorced/widowed & $1.22(0.28$ to 3.52$)$ & 0.750 \\
\hline Living with mother-in-law (yes/no) & $0.50(0.25$ to 0.90$)$ & 0.020 \\
\hline Job site of participants & & \\
$\quad$ Housewife or unemployed & Reference & 0.173 \\
\hline Work from home & $1.58(0.67$ to 3.26$)$ & 0.107 \\
\hline Employed & $0.86(0.62$ to 1.19$)$ & 0.007 \\
\hline Medical history of allergic rhinitis (yes/no) & $0.62(0.43$ to 0.88$)$ & 0.135 \\
\hline Health-related quality of life (physical functioning) & $0.98(0.96$ to 1.00$)$ & 0.002 \\
\hline Health-related quality of life (body pain) & $0.97(0.95$ to 0.98$)$ & 0.034 \\
\hline Exposed to secondary smoke during early pregnancy (daily/rarely) & $1.48(1.03$ to 2.11$)$ & \\
\hline Alcohol consumption & & 0.002 \\
\hline Drinker during early Pregnancy/never drunk & $0.34(0.14$ to 0.71$)$ & 0.027 \\
\hline Drinker during early pregnancy/ex-drinkers & $0.45(0.19$ to 0.92$)$ & \\
\hline Relationship with others & & $<0.001$ \\
\hline Visits to obstetrician (yes/no) & $0.02(0.02$ to 0.04$)$ & $<0.001$ \\
\hline Positive participation of partners (yes/no) & $0.26(0.18$ to 0.36$)$ & \\
\hline
\end{tabular}

For this model, data of 9298 people were used.

AlCc, 1427.9, LOF: $p=1.000$.

AIC, Akaike's Information Criteria; LOF, Lack Of Fit.

(35.9\% and $10.9 \%$, respectively). Macera and colleagues reported that responders were individuals who had family members with certain chronic conditions in their health-related survey. ${ }^{19}$ Leadbetter and colleagues examined the perceived risk of cancer by comparing early and late responders. They reported that the salience of the survey topic was associated with a prompt response. ${ }^{20}$ In this survey, participants with interest in children's allergic diseases were more likely to respond; however, daily exposure to secondary smoke made non-responses more likely.
In health-related surveys, participants with risky health behaviours are more likely to be non-respondents than are those who exhibit healthier behaviour. ${ }^{21}$

Etter and colleagues reported that respondents had better general health than did non-respondents. ${ }^{22}$ Martikainen and colleagues evaluated non-response bias in analyses of social class inequalities in health. ${ }^{23}$ They found that female non-respondents had an approximately 20\%-30\% higher sickness absence rate per 100 person-years than did respondents. Our results from the

Table 2B Multivariate logistic regression predicting the likelihood of survey non-response: model 2

\begin{tabular}{llc}
\hline Variable & OR (95\% Cl) & p value \\
\hline Living with mother-in-law (yes/no) & $0.47(0.24$ to 0.85$)$ & 0.011 \\
Having history of allergic rhinitis (yes/no) & $0.68(0.48$ to 0.95$)$ & 0.024 \\
Health-related quality of life (body pain) & $0.96(0.95$ to 0.98$)$ & $<0.001$ \\
Exposed to secondary smoke (daily/rarely) & $1.59(1.12$ to 2.23$)$ & 0.009 \\
Alcohol consumption (drinker/never drinker) & $0.36(0.16$ to 0.72$)$ & 0.002 \\
Alcohol consumption (drinker/ex-drinker) & $0.47(0.21$ to 0.92$)$ & 0.026 \\
Visits obstetrician (yes/no) & $0.02(0.02$ to 0.03$)$ & $<0.001$ \\
\hline Positive participation of participants' partners (yes/no) & $0.25(0.17$ to 0.35$)$ & $<0.001$ \\
\hline
\end{tabular}

For this analysis, data of 9634 people were used.

AICc, 1507.8, LOF: $p=1.000$.

AIC, Akaike's Information Criteria; LOF, Lack Of Fit. 
body pain scale showed that respondents were healthier than were non-respondents, which is consistent with these previous results. The response rate was higher among participants who lived with their mother-in-law, those who had partners who positively participated and those who maintained contact with an obstetrician. Alessi and colleagues suggested that general practitioners' understanding of the study could influence the attitude of their patients. ${ }^{24}$ Our results indicate that the same is true for people close to the participants. Hatta and colleagues reported that parents-in-law were perceived as the least cohesive persons among close family members in Japan. ${ }^{25}$ Another study of postpartum depression in China reported that the underlying cultural setting of the daughter-in-law/mother-in-law relationship contributed to depression among daughters-in-law. ${ }^{26}$ In our survey, the presence of a mother-in-law may have acted as a stressor to motivate the participants to return the questionnaires.

Furthermore, we collected participants' job status and categorised it into three modes: homemakers or unemployed, worked from home and employed. The response rate depended on participants' job, with a higher response rate being found among participants working from home than among those whose job location was outside of their home. In the Survey on Time Use and Leisure Activities in $2011,{ }^{27}$ women who worked from home (family workers) spent more time on housework and less time on self-education/training and hobbies/amusement than did those who were employed outside of their home. Associations with response to the questionnaire were also observed for job location and time spent answering the questionnaire; however, these relationships were weak.

Michikawa and colleagues reported that there was no difference in the distribution of maternal age at delivery between the JECS participants and the general population, further revealing that characteristics of selected infants in the JECS population (singleton birth, gestational age at birth, gender, birth weight) were similar to those of national survey data from the general population in Japan. ${ }^{2}$ The association between non-response and the relative factors found in this study was observed in Japanese pregnant women.

The limitations of this study are as follows: (1) a lack of information on education level and participants' socioeconomic status, (2) a lack of information on the survey mode and (3) a lack of information on partners' registration status. However, we know that socioeconomic status and education level are related to response status. ${ }^{62-30}$ In the JECS study, however, the socioeconomic and education status data were collected with the MT2 questionnaire, which was used to examine the non-response factor. Thus, we could not examine these factors. In particular, it seems that the investigators' interpretation of 'secondary smoke' was inconsistent with their results regarding alcohol consumption or health-related variables. These variables were related to socioeconomic and education status. In addition, several researchers have reported that response status differs according to survey mode. ${ }^{31-34}$ In this study, we collected questionnaires by hand or by mail. Because we were unable to collect data on the mode used, we did not evaluate the effect of these distinct modes. We were also unable to collect information regarding the extent of partners' participation-any response was considered positive. Finally, we could not confirm participants' medical or obstetric history using clinical data. Relying solely on data collected by self-administered questionnaires introduces the risk of response bias.

\section{CONCLUSIONS}

In conclusion, this study showed that obtaining understanding of the research objectives from people who are close to the participants was associated with a higher odds of response. To reduce the non-response rate in future follow-up surveys, additional efforts should be made to maintain contact and encourage participation among individuals who display relevant characteristics of potential non-responders. Because the data collected from pregnant women participating in JECS were used in this study, it means the participants may have been influenced by the Japanese culture and/or their socioeconomic situation. It is necessary to consider the results obtained from other participants from different cultures or nationalities.

\section{Author affiliations}

${ }^{1}$ Kanagawa University of Human Services, Yokosuka, Japan

${ }^{2}$ Department of Public Health, Faculty of Medicine, Graduate School of Medicine and Pharmaceutical Science for Education, University of Toyama, Toyama, Japan

${ }^{3}$ University of Toyama, Toyama Regional Center for JECS, Toyama, Japan

${ }^{4}$ Kanazawa University Health Service Center, Kanazawa, Japan

${ }^{5}$ Department of Obstetrics and Gynecology, Faculty of Medicine, Graduate School of Medicine and Pharmaceutical Science for Education, University of Toyama, Toyama, Japan

${ }^{6}$ Department of Pediatrics, Faculty of Medicine, Graduate School of Medicine and Pharmaceutical Science for Education, University of Toyama, Toyama, Japan ${ }^{7}$ Department of Biostatistics and Clinical Epidemiology, Faculty of Medicine, Graduate School of Medicine and Pharmaceutical Science for Education, University of Toyama, Toyama, Japan

Acknowledgements We thank all members of the Japan Environment and Children's Study (JECS), as of 2018.

Collaborators Members of the Japan Environment and Children's Study (JECS), as of 2018 (principal investigator, Toshihiro Kawamoto): Yukihiro Ohya (National Center for Child Health and Development, Tokyo, Japan), Reiko Kishi (Hokkaido Regional Center for JECS, Hokkaido University, Sapporo, Japan), Nobuo Yaegashi (Miyagi Regional Center for JECS, Tohoku University, Sendai, Japan), Koichi Hashimoto (Fukushima Regional Center for JECS, Fukushima Medical University, Fukushima, Japan), Chisato Mori (Chiba Regional Center for JECS, Chiba University, Chiba, Japan), Shuichi Ito (Kanagawa Regional Center for JECS, Yokohama City University, Yokohama, Japan), Zentaro Yamagata (Koshin Regional Center for JECS, University of Yamanashi, Chuo, Japan), Hidekuni Inadera (Toyama Regional Center for JECS, University of Toyama, Toyama, Japan), Michihiro Kamijima (Aichi Regional Center for JECS, Nagoya City University, Nagoya, Japan), Takeo Nakayama (Kyoto Regional Center for JECS, Kyoto University, Kyoto, Japan), Hiroyasu Iso (Osaka Regional Center for JECS, Osaka University, Suita, Japan), Masayuki Shima (Hyogo Regional Center for JECS, Hyogo College of Medicine, Nishinomiya, Japan), Yasuaki Hirooka (Tottori Regional Center for JECS, Tottori University, Yonago, Japan), Narufumi Suganuma (Kochi Regional Center for JECS, Kochi University, Nankoku, Japan), Koichi Kusuhara (Fukuoka Regional Center for JECS, University of Occupational and Environmental Health, Kitakyushu, Japan), and Takahiko Katoh (South Kyushu/ Okinawa Regional Center for JECS, Kumamoto University, Kumamoto, Japan). 
Contributors MK designed and conducted the study, performed the statistical analyses and wrote the manuscript. KM helped draft the manuscript. AT conducted the data collection and helped draft the manuscript. MI and TT conducted data collection and helped critically revise the manuscript. $\mathrm{KH}$ and $\mathrm{HI}$ participated in the study design and helped critically revise the manuscript. $\mathrm{HO}$ assisted with the statistical analyses. YA and SS helped critically revise the manuscript. All authors have read and approved the final manuscript.

Funding The Japan Environment and Children's Study was supported by the Ministry of the Environment, Japan. The findings and conclusions of this article are solely the responsibility of the authors and do not represent the official views of the above-mentioned government.

Competing interests None declared.

Patient consent for publication Obtained.

Provenance and peer review Not commissioned; externally peer reviewed.

Data sharing statement The dataset supporting the conclusions of this article will be available after the steering committee of the JECS allows it to become available. The dataset supporting the conclusions of this article is unsuitable for public deposition due to ethical restrictions and legal framework of Japan. The Act on the Protection of Personal Information (Act No. 57 of 30 May 2003; amendment on 9 September 2015) prohibits publicly depositing data containing personal information. Ethical Guidelines for Medical and Health Research Involving Human Subjects enforced by the Japan Ministry of Education, Culture, Sports, Science and Technology and the Ministry of Health, Labour and Welfare also restricts the open sharing of the epidemiological data. All inquiries about access to data should be sent to Dr Shoji F. Nakayama, JECS Programme Office, National Institute for Environmental Studies; email: jecs-en@nies.go.jp.

Open access This is an open access article distributed in accordance with the Creative Commons Attribution Non Commercial (CC BY-NC 4.0) license, which permits others to distribute, remix, adapt, build upon this work non-commercially, and license their derivative works on different terms, provided the original work is properly cited, appropriate credit is given, any changes made indicated, and the use is non-commercial. See: http://creativecommons.org/licenses/by-nc/4.0/.

\section{REFERENCES}

1. Kawamoto T, Nitta H, Murata K, et al. Rationale and study design of the Japan environment and children's study (JECS). BMC Public Health 2014;14:25.

2. Michikawa T, Nitta H, Nakayama SF, et al. The Japan Environment and Children's Study (JECS): A Preliminary Report on Selected Characteristics of Approximately 10000 Pregnant Women Recruited During the First Year of the Study. J Epidemiol 2015;25:452-8.

3. Christensen Al, Ekholm O, Gray L, et al. What is wrong with nonrespondents? Alcohol-, drug- and smoking-related mortality and morbidity in a 12-year follow-up study of respondents and nonrespondents in the Danish Health and Morbidity Survey. Addiction 2015;110:1505-12.

4. Schneider KL, Clark MA, Rakowski W, et al. Evaluating the impact of non-response bias in the Behavioral Risk Factor Surveillance System (BRFSS). J Epidemiol Community Health 2012;66:290-5.

5. Stang A. Nonresponse research-an underdeveloped field in epidemiology. Eur J Epidemiol 2003;18:929-32.

6. Pietilä $A M$, Rantakallio P, Läärä E. Background factors predicting non-response in a health survey of northern Finnish young men. Scand J Soc Med 1995;23:129-36.

7. Jackson R, Chambless LE, Yang K, et al. Differences between respondents and nonrespondents in a multicenter communitybased study vary by gender ethnicity. The Atherosclerosis Risk in Communities (ARIC) Study Investigators. J Clin Epidemiol 1996;49:1441-6.

8. Edwards P, Roberts I, Clarke M, et al. Increasing response rates to postal questionnaires: systematic review. BMJ 2002;324:1183.
9. Young AF, Powers JR, Bell SL. Attrition in longitudinal studies: who do you lose? Aust N Z J Public Health 2006;30:353-61.

10. Powers J, Loxton D. The impact of attrition in an 11-year prospective longitudinal study of younger women. Ann Epidemiol 2010;20:318-21.

11. Launes J, Hokkanen L, Laasonen M, et al. Attrition in a 30-year follow-up of a perinatal birth risk cohort: factors change with age. PeerJ 2014;2:e480.

12. Sheikh K, Mattingly S. Investigating non-response bias in mail surveys. J Epidemiol Community Health 1981;35:293-6.

13. Wolke D, Söhne B, Ohrt B, et al. Follow-up of preterm children: important to document dropouts. Lancet 1995;345:447.

14. Fukuhara S, Suzukamo Y. Manual of the SF-8 Japanese version. Kyoto, Japan: Institute for Health Outcomes \& Process Evaluation Research, 2004.

15. Furukawa TA, Kawakami N, Saitoh M, et al. The performance of the Japanese version of the K6 and K10 in the World Mental Health Survey Japan. Int J Methods Psychiatr Res 2008;17:152-8.

16. Baron R, Manniën J, te Velde SJ, et al. Socio-demographic inequalities across a range of health status indicators and health behaviours among pregnant women in prenatal primary care: a cross-sectional study. BMC Pregnancy Childbirth 2015;15:261.

17. Sakashita M, Hirota T, Harada M, et al. Prevalence of allergic rhinitis and sensitization to common aeroallergens in a Japanese population. Int Arch Allergy Immunol 2010;151:255-61.

18. Fernandez $C$. Ethical issues in health research in children. Paediatr Child Health 2008;13:707-12.

19. Macera CA, Jackson KL, Davis DR, et al. Patterns of non-response to a mail survey. J Clin Epidemiol 1990;43:1427-30.

20. Leadbetter S, Hawkins NA, Scholl LE, et al. Recruiting women for a study on perceived risk of cancer: influence of survey topic salience and early versus late response. Prev Chronic Dis 2013;10:E75.

21. Goldberg M, Chastang JF, Zins M, et al. Health problems were the strongest predictors of attrition during follow-up of the GAZEL cohort. J Clin Epidemiol 2006;59:1213-21.

22. Etter JF, Perneger TV. Analysis of non-response bias in a mailed health survey. J Clin Epidemiol 1997;50:1123-8.

23. Martikainen $P$, Laaksonen $M$, Piha $K$, et al. Does survey non-response bias the association between occupational social class and health? Scand J Public Health 2007;35:212-5.

24. Alessi D, Pastore G, Zuccolo L, et al. Analysis of nonresponse in the assessment of health-related quality of life of childhood cancer survivors. Eur J Cancer Prev 2007;16:576-80.

25. Hatta T, Kadobayashi S, Iwamoto T, et al. Interpersonal constructs of pregnant married women during pregnancy and postpartum period as perceptions of family members. Percept Mot Skills 2000;91(3 Pt 1):789-99.

26. Gao LL, Chan SW, You L, et al. Experiences of postpartum depression among first-time mothers in mainland China. $J$ Adv Nurs 2010;66:303-12.

27. Statistics Bureau, Ministry of Internal Affairs. Survey on time use and leisure activities. 2013/4/1 edn, 2013.

28. Helasoja V, Prättälä R, Dregval L, et al. Late response and item nonresponse in the Finbalt Health Monitor survey. Eur J Public Health 2002;12:117-23.

29. Tolonen H, Laatikainen T, Helakorpi S, et al. Marital status, educational level and household income explain part of the excess mortality of survey non-respondents. Eur J Epidemiol 2010;25:69-76.

30. Secor-Turner M, Sieving R, Widome R, et al. Active parent consent for health surveys with urban middle school students: processes and outcomes. J Sch Health 2010;80:73-9.

31. Bongers IM, Van Oers JA. Mode effects on self-reported alcohol use and problem drinking: mail questionnaires and personal interviewing compared. J Stud Alcohol 1998;59:280-5.

32. Tse A, Ching R, Ding Y-B, et al. Miscellany: A Comparison of the Effectiveness of Mail and Facsimile as Survey Media on Response Rate, Speed and Quality. Int J Market Res 1994;36:1-8.

33. Aquilino WS. Telephone versus face-to-face interviewing for household drug use surveys. Int J Addict 1992;27:71-91.

34. Picavet HS. National health surveys by mail or home interview: effects on response. J Epidemiol Community Health 2001;55:408-13. 